\title{
Kombination erhöht das Blutungsrisiko
}

Fragestellung: Beeinflusst die Begleitmedikation mit Thrombozytenfunktionshemmern bei Patienten mit Vorhofflimmern die Wirksamkeit und die Wahrscheinlichkeit von Blutungskomplikationen bei der Einnahme von Dabigatran oder Warfarin?

Hintergrund: Patienten mit Vorhofflimmern haben ein hohes Schlaganfallrisiko. Die wirksamste Form der Schlaganfallprävention ist eine orale Antikoagulation. Diese wurde bis vor kurzem mit Vitamin-K-Antagonisten wie Phenprocoumon oder Warfarin durchgeführt. Seit einiger Zeit stehen jetzt drei neue orale Antikoagulanzien zur Verfügung, die viele der Nachteile von Warfarin nicht haben. Seit langem ist bekannt, dass die Kombination von Thrombozytenfunktionshemmern mit Antikoagulanzien das Blutungsrisiko erhöht. Ob dies auch der Fall in der RE-LY-Studie ist, wurde in dieser Subgruppenanalyse untersucht.

Patienten und Methodik: Die RE-LY Studie war eine randomisierte Studie mit 18.113 Patienten mit Vorhofflimmern und mindestens einem vaskulärem Risikofaktor. Die Patienten wurden in drei Gruppen randomisiert und erhielten entweder Warfarin mit einer Ziel-INR von 2,0 bis 3,0 oder zweimal $110 \mathrm{mg}$ oder zweimal 150 mg Dabigatran. Der primäre Wirksamkeitsend-

Dans AL, Connolly SJ, Wallentin L et al. Concomitant use of antiplatelet therapy with dabigatran or warfarin in the randomized evaluation of long-term anticoagulation therapy (RE-LY) trial. Circulation $2013 ; 127$ : $634-40$ punkt war die Häufigkeit von Schlaganfall und systemischer Embolie und der primäre Sicherheitsendpunkt die Häufigkeit schwerwiegender Blutungskomplikationen. Im Laufe der Studie erhielten durchschnittlich $27 \%$ aller Patienten Thrombozy- tenfunktionshemmer, zumeist Acetylsalicylsäure und Clopidogrel.

Ergebnisse: Patienten die Thrombozytenfunktionshemmer erhielten hatten häufiger einen vorausgegangenen Myokardinfarkt, eine koronare Herzerkrankung, arterielle Hypertonie, einen CHADS-Score von mehr als drei und einen Diabetes mellitus. Die Wirksamkeit von Dabigatran zweimal 110 mg unterschied sich nicht in Abhängigkeit davon, ob Patienten Thrombozytenfunktionshemmer einnahmen oder nicht. Für beide Gruppen war Dabigatran nicht inferior im Vergleich zu Warfarin. Bei der hohen Dosis die wirksamer ist als die niedrige Dosis, betrug die relative Risikoreduktion für Schlaganfall und systemischer Embolien bei Patienten die keine Thrombozytenfunktionshemmer einnahmen $48 \%$ und für Patienten die Thrombozytenfunktionshemmer einnahmen nur $20 \%$. Die Einnahme von Thrombozytenfunktionshemmern erhöhte das Blutungsrisiko, wobei hier zwischen Warfarin und Dabigatran kein Unterschied bestand. Die Einnahme eines Thrombozytenfunktionshemmers zusätzlich zur Antikoagulation erhöhte das Risiko schwerwiegender Blutung um $60 \%$ und bei einer TripleTherapie mit zwei Thrombozytenfunktionshemmer stieg das Risiko um den Faktor 2,31. Das absolute Blutungsrisiko war erwartungsgemäß bei der niedrigen Dosis von Dabigatran geringer als bei der hohen Dosis oder bei Warfarin.

Schlussfolgerungen: Die Zugabe von Thrombozytenfunktionshemmern erniedrigt bei der hohen Dosis von Dabigatran im Vergleich zu Warfarin tendenziell die Wirksamkeit während die Rate an schwerwiegenden Blutungskomplikationen durch die Kombinationstherapie mit Thrombozytenfunktionshemmern steigt.

\section{-Kommentar von Hans-Christoph Diener, Essen}

\section{Kaum Nutzen, hohes Risiko}

Diese Subgruppenanalyse der RE-LY-Studie zeigt, dass eine Kombination von Antikoagulanzien und Thrombozytenfunktionshemmern das Blutungsrisiko erheblich steigert. Daher sollten Plättchenhemmer nur bei Patienten eingesetzt werden, bei denen eine klare Indikation besteht wie beispielsweise ein akutes Koronarsyndrom, ein Myokardinfarkt in den letzten zwölf Monaten oder bei Patienten, die einen Stent erhalten haben. Interessanterweise zeigt sich bei der hohen Dosis von Dabigatran ein ähnlicher Trend wie er früher bereits in den Ximelagatran-Studien beobachtet worden war, nämlich dass die Zugabe von Thrombozytenfunktionshemmern zur Antikoagulation die Risikoreduktion für vaskuläre Ereignisse abschwächt. Von besonderer Bedeutung ist die Beobachtung, dass Patienten mit stabiler koronarer Herzerkrankung von der Zugabe von Acetylsalicylsäure zur Antikoagulation nicht profitieren, sondern lediglich eine erhöhte Blutungsrate haben.

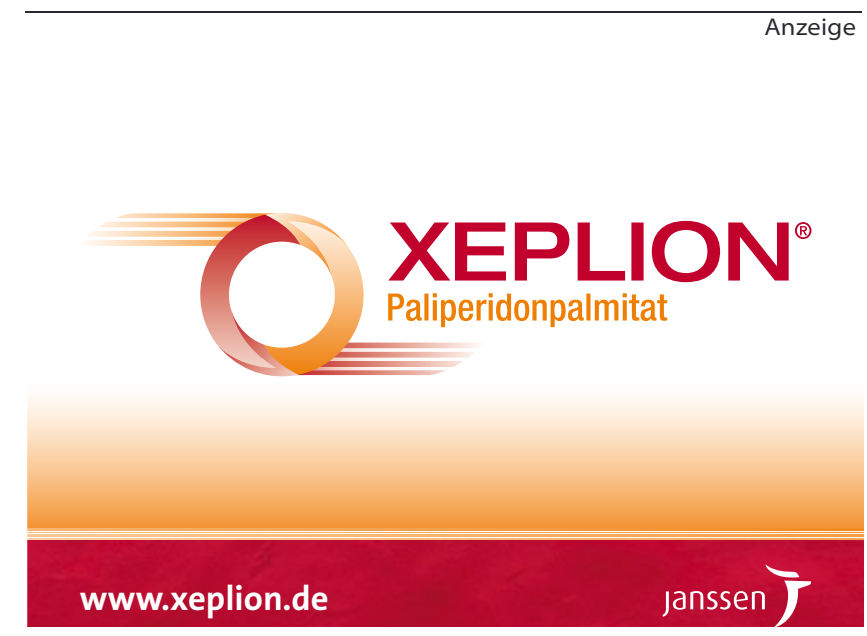

